\title{
Some Convergence Theorems for Contractive Type Mappings in CAT(0) Spaces
}

\author{
Kyung Soo Kim \\ Graduate School of Education, Mathematics Education, Kyungnam University, Changwon, Kyungnam 631-701, Republic of Korea \\ Correspondence should be addressed to Kyung Soo Kim; kksmj@kyungnam.ac.kr
}

Received 9 September 2013; Accepted 22 October 2013

Academic Editor: Sehie Park

Copyright (C) 2013 Kyung Soo Kim. This is an open access article distributed under the Creative Commons Attribution License, which permits unrestricted use, distribution, and reproduction in any medium, provided the original work is properly cited.

We establish theorems of strong convergence, for the Ishikawa-type (or two step; $c f$. Ishikawa, 1974) iteration scheme, to a fixed point of a uniformly $L$-Lipschitzian asymptotically demicontractive mapping and a uniformly $L$-Lipschitzian hemicontractive mapping in $C A T(0)$ space. Moreover, we will propose some open problems.

\section{Introduction}

Let $(X, d)$ be a metric space. One of the most interesting aspects of metric fixed point theory is to extend a linear version of known result to the nonlinear case in metric spaces. To achieve this, Takahashi [1] introduced a convex structure in a metric space $(X, d)$. A mapping $W: X \times X \times[0,1] \rightarrow X$ is a convex structure in $X$ if

$$
d(u, W(x, y, \lambda)) \leq \lambda d(u, x)+(1-\lambda) d(u, y)
$$

for all $x, y \in X$ and $\lambda \in[0,1]$. A metric space together with a convex structure $W$ is known as a convex metric space. A nonempty subset $K$ of a convex metric space is said to be convex if

$$
W(x, y, \lambda) \in K
$$

for all $x, y \in K$ and $\lambda \in[0,1]$. In fact, every normed space and its convex subsets are convex metric spaces but the converse is not true, in general (see, [1]).

Example 1 (see [2]). Let $X=\left\{\left(x_{1}, x_{2}\right) \in \mathbb{R}^{2}: x_{1}>0, x_{2}>0\right\}$, for all $x=\left(x_{1}, x_{2}\right), y=\left(y_{1}, y_{2}\right) \in X$, and $\lambda \in[0,1]$. We define a mapping $W: X \times X \times[0,1] \rightarrow X$ by

$$
W(x, y, \lambda)=\left(\lambda x_{1}+(1-\lambda) y_{1}, \frac{\lambda x_{1} x_{2}+(1-\lambda) y_{1} y_{2}}{\lambda x_{1}+(1-\lambda) y_{1}}\right),
$$

and define a metric $d: X \times X \rightarrow[0, \infty)$ by

$$
d(x, y)=\left|x_{1}-y_{1}\right|+\left|x_{1} x_{2}-y_{1} y_{2}\right| .
$$

Then we can show that $(X, d, W)$ is a convex metric space, but it is not a normed linear space.

A metric space $X$ is a $C A T(0)$ space (the term is due to Gromov [3] and it is an acronym for E. Cartan, A. D. Aleksandrov, and V. A. Toponogov) if it is geodesically connected and if every geodesic triangle in $X$ is at least as "thin" as its comparison triangle in the Euclidean plane (see, e.g., [4], page 159). It is well known that any complete, simply connected Riemannian manifold nonpositive sectional curvature is a $C A T(0)$ space. The precise definition is given below. For a thorough discussion of these spaces and of the fundamental role they play in various branches of mathematics, see Bridson and Haefliger [4] or Burago et al. [5].

Let $(X, d)$ be a metric space. A geodesic path joining $x \in X$ to $y \in X$ (or, more briefly, a geodesic from $x$ to $y$ ) is a mapping $c$ from a closed interval $[0, l] \subset \mathbb{R}$ to $X$ such that $c(0)=x$, $c(l)=y$, and $d\left(c(t), c\left(t^{\prime}\right)\right)=\left|t-t^{\prime}\right|$, for all $t, t^{\prime} \in[0, l]$. In particular, $c$ is an isometry and $d(x, y)=l$. The image $\alpha$ of $c$ is called a geodesic (or metric) segment joining $x$ and $y$. When it is unique, this geodesic is denoted by $[x, y]$. The space $(X, d)$ is said to be a geodesic space if every two points of $X$ are joined by a geodesic, and $X$ is said to be uniquely geodesic if there is exactly one geodesic joining $x$ and $y$ for each $x, y \in X$. A 
subset $Y \subseteq X$ is said to be convex if $Y$ includes every geodesic segment joining any two of its points.

A geodesic triangle $\Delta\left(x_{1}, x_{2}, x_{3}\right)$ is a geodesic metric space $(X, d)$ that consists of three points $x_{1}, x_{2}, x_{3} \in X$ (the vertices of $\Delta$ ) and is a geodesic segment between each pair of vertices (the edges of $\Delta$ ). A comparison triangle for the geodesic triangle $\Delta\left(x_{1}, x_{2}, x_{3}\right)$ in $(X, d)$ is a triangle $\bar{\Delta}\left(x_{1}, x_{2}, x_{3}\right)=$ $\Delta\left(\bar{x}_{1}, \bar{x}_{2}, \bar{x}_{3}\right)$ in $\mathbb{R}^{2}$ such that $d_{\mathbb{R}^{2}}\left(\bar{x}_{i}, \bar{x}_{j}\right)=d\left(x_{i}, x_{j}\right)$ for $i, j \in$ $\{1,2,3\}$. Such a triangle always exists (see, [4]).

A geodesic metric space is said to be a CAT(0) space if all geodesic triangles of appropriate size satisfy the following $C A T(0)$ comparison axiom.

Let $\Delta$ be a geodesic triangle in $X$ and let $\bar{\Delta} \subset \mathbb{R}^{2}$ be a comparison triangle for $\Delta$. Then $\Delta$ is said to satisfy the $C A T(0)$ inequality if for all $x, y \in \Delta$ and all comparison points $\bar{x}, \bar{y} \in \bar{\Delta}$,

$$
d(x, y) \leq d(\bar{x}, \bar{y})
$$

Complete CAT(0) spaces are often called Hadamard spaces (see, [6]). If $x, y_{1}, y_{2}$ are points of a CAT(0) space and if $y_{0}$ is the midpoint of the segment $\left[y_{1}, y_{2}\right]$, which we will denote by $y_{1} \oplus y_{2} / 2$, then the $\operatorname{CAT}(0)$ inequality implies

$$
d^{2}\left(x, \frac{y_{1} \oplus y_{2}}{2}\right) \leq \frac{1}{2} d^{2}\left(x, y_{1}\right)+\frac{1}{2} d^{2}\left(x, y_{2}\right)-\frac{1}{4} d^{2}\left(y_{1}, y_{2}\right)
$$

This inequality is the $(\mathrm{CN})$ inequality of Bruhat and Tits [7]. In fact, a geodesic space is a $C A T(0)$ space if and only if it satisfies the $(\mathrm{CN})$ inequality ( $c f$. [4], page 163). The previous inequality has been extended by Khamsi and Kirk [8] as

$$
\begin{aligned}
& d^{2}(z, \alpha x \oplus(1-\alpha) y) \\
& \quad \leq \alpha d^{2}(z, x)+(1-\alpha) d^{2}(z, y)-\alpha(1-\alpha) d^{2}(x, y),
\end{aligned}
$$

for any $\alpha \in[0,1]$ and $x, y, z \in X$. The inequality $\left(\mathrm{CN}^{*}\right)$ also appeared in [9].

Let us recall that a geodesic metric space is a $C A T(0)$ space if and only if it satisfies the (CN) inequality (see, [4], page 163). Moreover, if $X$ is a $C A T(0)$ metric space and $x, y \in X$, then for any $\alpha \in[0,1]$, there exists a unique point $\alpha x \oplus(1-\alpha) y \in$ $[x, y]$ such that

$$
d(z, \alpha x \oplus(1-\alpha) y) \leq \alpha d(z, x)+(1-\alpha) d(z, y)
$$

for any $z \in X$ and $[x, y]=\{\alpha x \oplus(1-\alpha) y: \alpha \in[0,1]\}$. In view of the previous inequality, $C A T(0)$ space has Takahashi's convex structure $W(x, y, \alpha)=\alpha x \oplus(1-\alpha) y$. It is easy to see that for any $x, y \in X$ and $\lambda \in[0,1]$,

$$
\begin{gathered}
d(x,(1-\lambda) x \oplus \lambda y)=\lambda d(x, y), \\
d(y,(1-\lambda) x \oplus \lambda y)=(1-\lambda) d(x, y) .
\end{gathered}
$$

As a consequence,

$$
\begin{gathered}
1 \cdot x \oplus 0 \cdot y=x, \\
(1-\lambda) x \oplus \lambda x=\lambda x \oplus(1-\lambda) x=x .
\end{gathered}
$$

Moreover, a subset $K$ of $C A T(0)$ space $X$ is convex if for any $x, y \in K$, we have $[x, y] \subset K$.

Definition 2. Let $C$ be a nonempty subset of a metric space $(X, d)$. Let $F(T)$ denote the fixed point set of $T$. Let $F(T) \neq \emptyset$.

(1) A mapping $T: C \rightarrow C$ is said to be $k$-strict asymptotically pseudocontractive with sequence $\left\{a_{n}\right\}$ if $\lim _{n \rightarrow \infty} a_{n}=1$ for some constant $k, 0 \leq k<1$ and

$$
d^{2}\left(T^{n} x, T^{n} y\right) \leq a_{n}^{2} d^{2}(x, y)+k\left(d\left(x, T^{n} x\right)-d\left(y, T^{n} y\right)\right)^{2},
$$

for all $x, y \in C, n \in \mathbb{N}$.

If $k=0$, then $T$ is said to be asymptotically nonexpansive with sequence $\left\{a_{n}\right\}$, that is,

$$
d\left(T^{n} x, T^{n} y\right) \leq a_{n} d(x, y), \quad \forall x, y \in C .
$$

(2) A mapping $T: C \rightarrow C$ is said to be asymptotically demicontractive with sequence $\left\{a_{n}\right\}$ if $\lim _{n \rightarrow \infty} a_{n}=1$ for some constant $k, 0 \leq k<1$, and

$$
\begin{array}{r}
d^{2}\left(T^{n} x, p\right) \leq a_{n}^{2} d^{2}(x, p)+k \cdot d^{2}\left(x, T^{n} x\right), \\
\forall p \in F(T),
\end{array}
$$

for all $x \in C, n \in \mathbb{N}$.

If $k=0$, then $T$ is said to be asymptotically quasinonexpansive with sequence $\left\{a_{n}\right\}$, that is,

$$
d\left(T^{n} x, p\right) \leq a_{n} d(x, p), \quad \forall x \in C, \forall p \in F(T) .
$$

(3) A mapping $T: C \rightarrow C$ is said to be asymptotically pseudocontractive with sequence $\left\{a_{n}\right\}$ if $\lim _{n \rightarrow \infty} a_{n}=$ 1 and

$$
d^{2}\left(T^{n} x, T^{n} y\right) \leq a_{n} d^{2}(x, y)+\left[d\left(x, T^{n} x\right)-d\left(y, T^{n} y\right)\right]^{2}
$$

for all $x, y \in C, n \in \mathbb{N}$.

(4) A mapping $T: C \rightarrow C$ is said to be asymptotically hemicontractive with sequence $\left\{a_{n}\right\}$ if $\lim _{n \rightarrow \infty} a_{n}=1$ and

$$
d^{2}\left(T^{n} x, p\right) \leq a_{n} d^{2}(x, p)+d^{2}\left(x, T^{n} x\right), \quad \forall p \in F(T),
$$

for all $x \in C, n \in \mathbb{N}$.

(5) A mapping $T: C \rightarrow C$ is said to be uniformly $L$ Lipschitzian if for some constant $L>0$,

$$
d\left(T^{n} x, T^{n} y\right) \leq L \cdot d(x, y), \quad \forall x, y \in C,
$$

for all $n \in \mathbb{N}$.

Liu [10] has proved the convergence of Mann and Ishikawa iterative sequence for uniformly $L$-Lipschitzian asymptotically demicontractive and hemicontractive mappings in Hilbert space ( $c f$. [11]). The existence of (common) 
fixed points of one mapping (or two mappings or family of mappings) is not known in many situations. So the approximation of fixed points of one or more nonexpansive, asymptotically nonexpansive, or asymptotically quasinonexpansive mappings by various iterations have been extensively studied in Banach spaces, convex metric spaces, CAT(0) spaces, and so on (see, $[2,6,8,9,12-27])$.

In this paper, we establish theorems of strong convergence for the Ishikawa-type (or two step, $c f$. [28]) iteration scheme to a fixed point of a uniformly $L$-Lipschitzian asymptotically demicontractive mapping and a uniformly L-Lipschitzian asymptotically hemicontractive mapping in $C A T(0)$ space. Moreover, we will propose some open problems.

\section{Preliminaries}

We introduce the following iteration process.

Let $C$ be a nonempty convex subset of a $C A T(0)$ space $(X, d)$ and let $T: C \rightarrow C$ be a given mapping. Let $x_{1} \in C$ be a given point.

Algorithm 3. The sequences $\left\{x_{n}\right\}$ and $\left\{y_{n}\right\}$ defined by the iterative process

$$
\begin{gathered}
x_{n+1}=\left(1-\alpha_{n}\right) x_{n} \oplus \alpha_{n} T^{n} y_{n}, \\
y_{n}=\left(1-\beta_{n}\right) x_{n} \oplus \beta_{n} T^{n} x_{n}, \quad n \geq 1,
\end{gathered}
$$

is called an Ishikawa-type iterative sequence (cf. [28]).

If $\beta_{n} \equiv 0$, then Algorithm 3 reduces to the following.

Algorithm 4. The sequence $\left\{x_{n}\right\}$ defined by the iterative process

$$
x_{n+1}=\left(1-\alpha_{n}\right) x_{n} \oplus \alpha_{n} T^{n} x_{n}, \quad n \geq 1,
$$

is called a Mann-type iterative sequence (cf. [29]).

Lemma 5 (see [10]). Let sequences $\left\{a_{n}\right\},\left\{b_{n}\right\}$ satisfy that

$$
a_{n+1} \leq a_{n}+b_{n}
$$

$a_{n} \geq 0$, for all $n \in \mathbb{N}, \sum_{n=1}^{\infty} b_{n}$ is convergent, and $\left\{a_{n}\right\}$ has a subsequence $\left\{a_{n_{k}}\right\}$ converging to 0 . Then, we must have

$$
\lim _{n \rightarrow \infty} a_{n}=0
$$

\section{Convergence Theorems}

Lemma 6. Let $(X, d)$ be a $C A T(0)$ space and let $C$ be a nonempty convex subset of $X$. Let $T: C \rightarrow C$ be an uniformly L-Lipschitzian mapping and let $\left\{\alpha_{n}\right\},\left\{\beta_{n}\right\}$ be sequence in $[0,1]$. Define the iteration scheme $\left\{x_{n}\right\}$ as Algorithm 3. Then

$$
\begin{aligned}
d\left(x_{n}, T x_{n}\right) \leq & d\left(x_{n}, T^{n} x_{n}\right) \\
& +L\left(1+2 L+L^{2}\right) d\left(x_{n-1}, T^{n-1} x_{n-1}\right),
\end{aligned}
$$

for all $n \geq 1$.
Proof. Let $C_{n}=d\left(x_{n}, T^{n} x_{n}\right)$. We have

$$
\begin{aligned}
d\left(x_{n-1}, y_{n-1}\right) & =d\left(x_{n-1},\left(1-\beta_{n-1}\right) x_{n-1} \oplus \beta_{n-1} T^{n-1} x_{n-1}\right) \\
& \leq \beta_{n-1} \cdot d\left(x_{n-1}, T^{n-1} x_{n-1}\right) \\
& =\beta_{n-1} C_{n-1} .
\end{aligned}
$$

From (22), we get

$$
\begin{aligned}
d\left(x_{n-1}, T^{n-1} y_{n-1}\right) \leq & d\left(x_{n-1}, T^{n-1} x_{n-1}\right) \\
& +d\left(T^{n-1} x_{n-1}, T^{n-1} y_{n-1}\right) \\
\leq & C_{n-1}+L \cdot d\left(x_{n-1}, y_{n-1}\right) \\
\leq & C_{n-1}+\beta_{n-1} \cdot L \cdot C_{n-1} .
\end{aligned}
$$

From (22) and (23), we get

$$
\begin{aligned}
d\left(x_{n}, T x_{n}\right) & \leq d\left(x_{n}, T^{n} x_{n}\right)+d\left(T^{n} x_{n}, T x_{n}\right) \\
\leq & C_{n}+L \cdot d\left(T^{n-1} x_{n}, x_{n}\right) \\
\leq & C_{n}+L\left\{d\left(T^{n-1} x_{n}, T^{n-1} x_{n-1}\right)+d\left(T^{n-1} x_{n-1}, x_{n}\right)\right\} \\
\leq & C_{n}+L^{2} \cdot d\left(x_{n}, x_{n-1}\right)+L \cdot d\left(T^{n-1} x_{n-1}, x_{n}\right) \\
\leq & C_{n}+L^{2} \cdot d\left(\left(1-\alpha_{n-1}\right) x_{n-1} \oplus \alpha_{n-1} T^{n-1} y_{n-1}, x_{n-1}\right) \\
& +L \cdot d\left(T^{n-1} x_{n-1},\left(1-\alpha_{n-1}\right) x_{n-1} \oplus \alpha_{n-1} T^{n-1} y_{n-1}\right) \\
\leq & C_{n}+L^{2} \cdot \alpha_{n-1} \cdot d\left(T^{n-1} y_{n-1}, x_{n-1}\right) \\
& +L\left\{\left(1-\alpha_{n-1}\right) d\left(T^{n-1} x_{n-1}, x_{n-1}\right)\right. \\
& \left.\quad+\alpha_{n-1} \cdot d\left(T^{n-1} x_{n-1}, T^{n-1} y_{n-1}\right)\right\} \\
\leq & C_{n}+L^{2} \cdot \alpha_{n-1}\left(C_{n-1}+\beta_{n-1} \cdot L \cdot C_{n-1}\right) \\
& +L\left(1-\alpha_{n-1}\right) C_{n-1}+L^{2} \cdot \alpha_{n-1} \cdot \beta_{n-1} \cdot C_{n-1} \\
\leq & C_{n}+L\left(1+2 L+L^{2}\right) C_{n-1}, n \geq 1 .
\end{aligned}
$$

This completes the proof of Lemma 6.

Theorem 7. Let $(X, d)$ be a complete $C A T(0)$ space, let $C$ be a nonempty bounded closed convex subset of $X$, and let $T: C \rightarrow C$ be a completely continuous and uniformly $L$ Lipschitzian and asymptotically demicontractive with sequence $\left\{a_{n}\right\}, a_{n} \in[1, \infty), \sum_{n=1}^{\infty}\left(a_{n}^{2}-1\right)<\infty, \varepsilon \leq \alpha_{n} \leq 1-k-\varepsilon$, for all $n \in \mathbb{N}$ and some $\varepsilon>0$. Given $x_{0} \in C$, define the iteration scheme $\left\{x_{n}\right\}$ by

$$
x_{n+1}=\left(1-\alpha_{n}\right) x_{n} \oplus \alpha_{n} T^{n} x_{n}, \quad n \geq 1 .
$$

Then $\left\{x_{n}\right\}$ converges strongly to some fixed point of $T$. 
Proof. Since $T$ is a completely continuous mapping in a bounded closed convex subset $C$ of complete metric space, from Schauder's theorem, $F(T)$ is nonempty. It follows from $\left(\mathrm{CN}^{*}\right)$ inequality that

$$
\begin{aligned}
d^{2}\left(x_{n+1}, p\right)= & d^{2}\left(\left(1-\alpha_{n}\right) x_{n} \oplus \alpha_{n} T^{n} x_{n}, p\right) \\
\leq & \left(1-\alpha_{n}\right) d^{2}\left(x_{n}, p\right)+\alpha_{n} d^{2}\left(T^{n} x_{n}, p\right) \\
& -\left(1-\alpha_{n}\right) \alpha_{n} d^{2}\left(x_{n}, T^{n} x_{n}\right),
\end{aligned}
$$

for all $p \in F(T)$. Since $T$ is a asymptotically demicontractive, we get

$$
\begin{aligned}
& d^{2}\left(x_{n+1}, p\right) \leq\left(1-\alpha_{n}\right) d^{2}\left(x_{n}, p\right) \\
&+\alpha_{n}\left\{a_{n}^{2} d^{2}\left(x_{n}, p\right)+k \cdot d^{2}\left(x_{n}, T^{n} x_{n}\right)\right\} \\
&-\alpha_{n}\left(1-\alpha_{n}\right) d^{2}\left(x_{n}, T^{n} x_{n}\right) \\
&= d^{2}\left(x_{n}, p\right)+\alpha_{n}\left(a_{n}^{2}-1\right) d^{2}\left(x_{n}, p\right) \\
&-\alpha_{n}\left(1-\alpha_{n}-k\right) d^{2}\left(x_{n}, T^{n} x_{n}\right), \\
& \forall p \in F(T) .
\end{aligned}
$$

Since $0<\varepsilon \leq \alpha_{n} \leq 1-k-\varepsilon$, we have $1-k-\alpha_{n} \geq \varepsilon$. Thus,

$$
\alpha_{n}\left(1-k-\alpha_{n}\right) \geq \varepsilon^{2} .
$$

From (27), we have

$$
\begin{aligned}
d^{2}\left(x_{n+1}, p\right) \leq & d^{2}\left(x_{n}, p\right)+\alpha_{n}\left(a_{n}^{2}-1\right) d^{2}\left(x_{n}, p\right) \\
& -\varepsilon^{2} \cdot d^{2}\left(x_{n}, T^{n} x_{n}\right),
\end{aligned}
$$

for all $p \in F(T)$. Since $C$ is bounded and $T$ is self-mapping in $C$, there exist some $M>0$ so that $d^{2}\left(x_{n}, p\right) \leq M$, for all $n \in \mathbb{N}$. Since $0 \leq \alpha_{n} \leq 1$, it follows from (29) that

$$
\begin{array}{r}
d^{2}\left(x_{n+1}, p\right) \leq d^{2}\left(x_{n}, p\right)+\left(a_{n}^{2}-1\right) M-\varepsilon^{2} \cdot d^{2}\left(x_{n}, T^{n} x_{n}\right) \\
\forall p \in F(T) .
\end{array}
$$

Therefore,

$$
\varepsilon^{2} \cdot d^{2}\left(x_{n}, T^{n} x_{n}\right) \leq d^{2}\left(x_{n}, p\right)-d^{2}\left(x_{n+1}, p\right)+\left(a_{n}^{2}-1\right) M .
$$

So

$$
\begin{aligned}
& \sum_{n=1}^{m} \varepsilon^{2} \cdot d^{2}\left(x_{n}, T^{n} x_{n}\right) \\
& \quad \leq d^{2}\left(x_{1}, p\right)-d^{2}\left(x_{m+1}, p\right)+\sum_{n=1}^{m}\left(a_{n}^{2}-1\right) M \\
& \quad \leq 2 M+\sum_{n=1}^{\infty}\left(a_{n}^{2}-1\right) M,
\end{aligned}
$$

for all $m \in \mathbb{N}$. Since $\sum_{n=1}^{\infty}\left(a_{n}^{2}-1\right)<\infty$, we get

$$
\sum_{n=1}^{\infty} \varepsilon^{2} \cdot d^{2}\left(x_{n}, T^{n} x_{n}\right)<\infty .
$$

Therefore,

$$
\lim _{n \rightarrow \infty} d^{2}\left(x_{n}, T^{n} x_{n}\right)=0, \quad \lim _{n \rightarrow \infty} d\left(x_{n}, T^{n} x_{n}\right)=0 .
$$

Since $T$ is a uniformly $L$-Lipschitzian, it follows from Lemma 6 that

$$
\lim _{n \rightarrow \infty} d\left(x_{n}, T x_{n}\right)=0 .
$$

Since $\left\{x_{n}\right\}$ is a bounded sequence and $T$ is completely continuous, there exist a convergent subsequence $\left\{T x_{n_{k}}\right\}$ of $\left\{T x_{n}\right\}$. Therefore, from (35), $\left\{x_{n}\right\}$ has a convergent subsequence $\left\{x_{n_{k}}\right\}$. Let $\lim _{k \rightarrow \infty} x_{n_{k}}=q$. It follows from the continuity of $T$ and (35), we have $q=T q$. Therefore, $\left\{x_{n}\right\}$ has a subsequence which converges to the fixed point $q$ of $T$. Let $p=q$ in the inequality (30). Since $\sum_{n=1}^{\infty}\left(a_{n}^{2}-1\right)<\infty$ and $\sum_{n=1}^{\infty} \varepsilon^{2}$. $d^{2}\left(x_{n}, T^{n} x_{n}\right)<\infty$, from (30) and Lemma 5, we have

$$
\lim _{n \rightarrow \infty} d^{2}\left(x_{n}, q\right)=0 .
$$

Therefore,

$$
\lim _{n \rightarrow \infty} x_{n}=q
$$

This completes the proof of Theorem 7 .

Corollary 8. Let $(X, d)$ be a complete CAT $(0)$ space, let $C$ be a nonempty bounded closed convex subset of $X$, and let $T: C \rightarrow$ $C$ be a completely continuous and uniformly L-Lipschitzian and $k$-strict asymptotically pseudocontractive with sequence $\left\{a_{n}\right\}$, $a_{n} \in[1, \infty), \sum_{n=1}^{\infty}\left(a_{n}^{2}-1\right)<\infty$, and $\varepsilon \leq \alpha_{n} \leq 1-k-\varepsilon$, for all $n \in \mathbb{N}$ and some $\varepsilon>0$. Given $x_{0} \in C$, define the iteration scheme $\left\{x_{n}\right\}$ by

$$
x_{n+1}=\left(1-\alpha_{n}\right) x_{n} \oplus \alpha_{n} T^{n} x_{n}, \quad n \geq 1 .
$$

Then $\left\{x_{n}\right\}$ converges strongly to some fixed point of T.

Proof. By Definition 2, $T$ is $k$-strict asymptotically pseudocontractive; then $T$ must be asymptotically demicontractive. Therefore, Corollary 8 can be proved by using Theorem 7.

Lemma 9. Let $(X, d)$ be a $C A T(0)$ space and let $C$ be a nonempty convex subset of $X$. Let $T: C \rightarrow C$ be an uniformly L-Lipschitzian and asymptotically hemicontractive with sequence $\left\{a_{n}\right\} \subset[1, \infty)$, for all $n \in \mathbb{N}$, and $F(T)$ is nonempty. Define the iteration scheme $\left\{x_{n}\right\}$ as follows:

$$
\begin{gathered}
x_{n+1}=\left(1-\alpha_{n}\right) x_{n} \oplus \alpha_{n} T^{n} y_{n}, \\
y_{n}=\left(1-\beta_{n}\right) x_{n} \oplus \beta_{n} T^{n} x_{n}, \quad n \geq 1 .
\end{gathered}
$$

Then the following inequality holds:

$$
\begin{aligned}
d^{2}\left(x_{n+1}, p\right) \leq & {\left[1+\alpha_{n}\left(a_{n}-1\right)\left(a_{n} \beta_{n}+1\right)\right] d^{2}\left(x_{n}, p\right) } \\
& -\alpha_{n} \beta_{n}\left(1-\beta_{n}-a_{n} \beta_{n}-L^{2} \beta_{n}^{2}\right) d^{2}\left(x_{n}, T^{n} x_{n}\right) \\
& -\alpha_{n}\left(\beta_{n}-\alpha_{n}\right) d^{2}\left(x_{n}, T^{n} y_{n}\right),
\end{aligned}
$$

for all $p \in F(T)$. 
Proof. It follows from $\left(\mathrm{CN}^{*}\right)$ inequality that

$$
\begin{aligned}
d^{2}\left(x_{n+1}, p\right)= & d^{2}\left(\left(1-\alpha_{n}\right) x_{n} \oplus \alpha_{n} T^{n} y_{n}, p\right) \\
\leq & \left(1-\alpha_{n}\right) d^{2}\left(x_{n}, p\right)+\alpha_{n} d^{2}\left(T^{n} y_{n}, p\right) \\
& -\left(1-\alpha_{n}\right) \alpha_{n} d^{2}\left(x_{n}, T^{n} y_{n}\right), \\
d^{2}\left(y_{n}, p\right)= & d^{2}\left(\left(1-\beta_{n}\right) x_{n} \oplus \beta_{n} T^{n} x_{n}, p\right) \\
\leq & \left(1-\beta_{n}\right) d^{2}\left(x_{n}, p\right)+\beta_{n} d^{2}\left(T^{n} x_{n}, p\right) \\
- & \left(1-\beta_{n}\right) \beta_{n} d^{2}\left(x_{n}, T^{n} x_{n}\right),
\end{aligned}
$$

for all $p \in F(T)$. Since $T$ is asymptotically hemicontractive, we get

$$
\begin{gathered}
d^{2}\left(T^{n} y_{n}, p\right) \leq a_{n} d^{2}\left(y_{n}, p\right)+d^{2}\left(y_{n}, T^{n} y_{n}\right), \\
d^{2}\left(T^{n} x_{n}, p\right) \leq a_{n} d^{2}\left(x_{n}, p\right)+d^{2}\left(x_{n}, T^{n} x_{n}\right) .
\end{gathered}
$$

From (42) and (44), we have

$$
\begin{aligned}
d^{2}\left(y_{n}, p\right) \leq & \left(1-\beta_{n}\right) d^{2}\left(x_{n}, p\right) \\
& +\beta_{n}\left[a_{n} d^{2}\left(x_{n}, p\right)+d^{2}\left(x_{n}, T^{n} x_{n}\right)\right] \\
& -\left(1-\beta_{n}\right) \beta_{n} d^{2}\left(x_{n}, T^{n} x_{n}\right) \\
= & {\left[1+\left(a_{n}-1\right) \beta_{n}\right] d^{2}\left(x_{n}, p\right)+\beta_{n}^{2} d^{2}\left(x_{n}, T^{n} x_{n}\right) . }
\end{aligned}
$$

From $\left(\mathrm{CN}^{*}\right)$ inequality, we have

$$
\begin{aligned}
d^{2}\left(y_{n}, T^{n} y_{n}\right)= & d^{2}\left(\left(1-\beta_{n}\right) x_{n} \oplus \beta_{n} T^{n} x_{n}, T^{n} y_{n}\right) \\
\leq & \left(1-\beta_{n}\right) d^{2}\left(x_{n}, T^{n} y_{n}\right)+\beta_{n} d^{2}\left(T^{n} x_{n}, T^{n} y_{n}\right) \\
& -\left(1-\beta_{n}\right) \beta_{n} d^{2}\left(x_{n}, T^{n} x_{n}\right)
\end{aligned}
$$

Substituting (45) and (46) into (43), we get

$$
\begin{aligned}
d^{2}( & \left.T^{n} y_{n}, p\right) \\
\leq & a_{n}\left[1+\left(a_{n}-1\right) \beta_{n}\right] d^{2}\left(x_{n}, p\right)+a_{n} \beta_{n}^{2} d^{2}\left(x_{n}, T^{n} x_{n}\right) \\
& +\left(1-\beta_{n}\right) d^{2}\left(x_{n}, T^{n} y_{n}\right)+\beta_{n} d^{2}\left(T^{n} x_{n}, T^{n} y_{n}\right) \\
& -\left(1-\beta_{n}\right) \beta_{n} d^{2}\left(x_{n}, T^{n} x_{n}\right) .
\end{aligned}
$$

From (41) and (47), we obtain

$$
\begin{aligned}
d^{2}( & \left.x_{n+1}, p\right) \\
\leq & \left(1-\alpha_{n}\right) d^{2}\left(x_{n}, p\right)+\alpha_{n} a_{n}\left(1+\left(a_{n}-1\right) \beta_{n}\right) d^{2}\left(x_{n}, p\right) \\
& +\alpha_{n} a_{n} \beta_{n}^{2} d^{2}\left(x_{n}, T^{n} x_{n}\right)+\alpha_{n}\left(1-\beta_{n}\right) d^{2}\left(x_{n}, T^{n} y_{n}\right) \\
& +\alpha_{n} \beta_{n} d^{2}\left(T^{n} x_{n}, T^{n} y_{n}\right)-\alpha_{n}\left(1-\beta_{n}\right) \beta_{n} d^{2}\left(x_{n}, T^{n} x_{n}\right) \\
& -\left(1-\alpha_{n}\right) \alpha_{n} d^{2}\left(x_{n}, T^{n} y_{n}\right) \\
= & {\left[1+\alpha_{n}\left\{\left(a_{n}-1\right)+a_{n}\left(a_{n}-1\right) \beta_{n}\right\}\right] d^{2}\left(x_{n}, p\right) } \\
& -\alpha_{n} \beta_{n}\left(1-\beta_{n}-a_{n} \beta_{n}\right) d^{2}\left(x_{n}, T^{n} x_{n}\right) \\
& -\alpha_{n}\left(\beta_{n}-\alpha_{n}\right) d^{2}\left(x_{n}, T^{n} y_{n}\right)+\alpha_{n} \beta_{n} d^{2}\left(T^{n} x_{n}, T^{n} y_{n}\right) .
\end{aligned}
$$

Since $T$ is uniformly $L$-Lipschitzian, we have

$$
\begin{aligned}
d\left(T^{n} x_{n}, T^{n} y_{n}\right) & \leq L \cdot d\left(x_{n}, y_{n}\right) \\
& =L \cdot d\left(x_{n},\left(1-\beta_{n}\right) x_{n} \oplus \beta_{n} T^{n} x_{n}\right) \\
& \leq L \beta_{n} \cdot d\left(x_{n}, T^{n} x_{n}\right)
\end{aligned}
$$

Substituting (49) into (48), we obtain

$$
\begin{aligned}
d^{2}\left(x_{n+1}, p\right) \leq & {\left[1+\alpha_{n}\left(a_{n}-1\right)\left(1+a_{n} \beta_{n}\right)\right] d^{2}\left(x_{n}, p\right) } \\
& -\alpha_{n} \beta_{n}\left(1-\beta_{n}-a_{n} \beta_{n}-L^{2} \beta_{n}^{2}\right) d^{2}\left(x_{n}, T^{n} x_{n}\right) \\
& -\alpha_{n}\left(\beta_{n}-\alpha_{n}\right) d^{2}\left(x_{n}, T^{n} y_{n}\right), \quad \forall p \in F(T) .
\end{aligned}
$$

This completes the proof of Lemma 9.

Lemma 10. Let $(X, d)$ be a $C A T(0)$ space and let $C$ be a nonempty bounded convex subset of $X$. Let $T: C \rightarrow C$ be a uniformly L-Lipschitzian and asymptotically hemicontractive with sequence $\left\{a_{n}\right\} \subset[1, \infty)$, for all $n \in \mathbb{N}$ and $\sum_{n=1}^{\infty}\left(a_{n}-1\right)<$ $\infty$. Let $F(T)$ be nonempty. Given $x_{1} \in C$, define the iteration scheme $\left\{x_{n}\right\}$ by

$$
\begin{gathered}
x_{n+1}=\left(1-\alpha_{n}\right) x_{n} \oplus \alpha_{n} T^{n} y_{n}, \\
y_{n}=\left(1-\beta_{n}\right) x_{n} \oplus \beta_{n} T^{n} x_{n}, \quad n \geq 1 .
\end{gathered}
$$

If $\varepsilon \leq \alpha_{n} \leq \beta_{n} \leq b$ for some $\varepsilon>0$ and $b \in\left(0,\left(\sqrt{1+L^{2}}-1\right) / L^{2}\right)$, then

$$
\lim _{n \rightarrow \infty} d\left(x_{n}, T x_{n}\right)=0 .
$$

Proof. First, we will prove $\lim _{n \rightarrow \infty} d\left(x_{n}, T^{n} x_{n}\right)=0$. From Lemma 9 and $0 \leq \alpha_{n} \leq \beta_{n}$, we have

$$
\begin{aligned}
d^{2}\left(x_{n+1}, p\right) \leq & {\left[1+\alpha_{n}\left(a_{n}-1\right)\left(1+a_{n} \beta_{n}\right)\right] d^{2}\left(x_{n}, p\right) } \\
& -\alpha_{n} \beta_{n}\left(1-\beta_{n}-a_{n} \beta_{n}-L^{2} \beta_{n}^{2}\right) d^{2}\left(x_{n}, T^{n} x_{n}\right) .
\end{aligned}
$$


Thus

$$
\begin{aligned}
& d^{2}\left(x_{n+1}, p\right)-d^{2}\left(x_{n}, p\right) \\
& \quad \leq \alpha_{n}\left(a_{n}-1\right)\left(1+a_{n} \beta_{n}\right) d^{2}\left(x_{n}, p\right) \\
& \quad-\alpha_{n} \beta_{n}\left(1-\beta_{n}-a_{n} \beta_{n}-L^{2} \beta_{n}^{2}\right) d^{2}\left(x_{n}, T^{n} x_{n}\right) .
\end{aligned}
$$

Since $\sum_{n=1}^{\infty}\left(a_{n}-1\right)<\infty$, we have $\lim _{n \rightarrow \infty}\left(a_{n}-1\right)=0$. Hence, $\left\{a_{n}\right\}$ is bounded. By boundedness of $C$ and $0 \leq \alpha_{n} \leq \beta_{n} \leq 1$, we obtain that $\left\{\alpha_{n}\left(1+a_{n} \beta_{n}\right) d^{2}\left(x_{n}, p\right)\right\}$ is bounded. Therefore, there exists a constant $M>0$ such that

$$
0 \leq \alpha_{n}\left(1+a_{n} \beta_{n}\right) d^{2}\left(x_{n}, p\right) \leq M .
$$

From (54) and (55), we get

$$
\begin{aligned}
& d^{2}\left(x_{n+1}, p\right)-d^{2}\left(x_{n}, p\right) \\
& \leq\left(a_{n}-1\right) M-\alpha_{n} \beta_{n}\left(1-\beta_{n}-a_{n} \beta_{n}\right. \\
& \left.\quad-L^{2} \beta_{n}^{2}\right) d^{2}\left(x_{n}, T^{n} x_{n}\right) .
\end{aligned}
$$

Let $D=1-2 b-L^{2} b^{2}>0$. Since $\lim _{n \rightarrow \infty} a_{n}=1$, there exists $N \in \mathbb{N}$ such that

$$
1-\beta_{n}-a_{n} \beta_{n}-L^{2} \beta_{n}^{2} \geq 1-b-a_{n} b-L^{2} b^{2} \geq \frac{D}{2}>0,
$$

for all $n \geq N$. Suppose that $\lim _{n \rightarrow \infty} d\left(x_{n}, T^{n} x_{n}\right) \neq 0$, then there exist a $\varepsilon_{0}>0$ and a subsequence $\left\{x_{n_{i}}\right\}$ of $\left\{x_{n}\right\}$ such that

$$
d^{2}\left(x_{n_{i}}, T^{n_{i}} x_{n_{i}}\right) \geq \varepsilon_{0} .
$$

Without loss of generality, we let $n_{1} \geq N$. From (56), we have

$$
\begin{aligned}
\alpha_{n} \beta_{n} & \left(1-\beta_{n}-a_{n} \beta_{n}-L^{2} \beta_{n}^{2}\right) d^{2}\left(x_{n}, T^{n} x_{n}\right) \\
\leq & \left(a_{n}-1\right) M+d^{2}\left(x_{n}, p\right)-d^{2}\left(x_{n+1}, p\right),
\end{aligned}
$$

so

$$
\begin{aligned}
& \sum_{l=1}^{i} \alpha_{n_{l}} \beta_{n_{l}}\left(1-\beta_{n_{l}}-a_{n_{l}} \beta_{n_{l}}-L^{2} \beta_{n_{l}}^{2}\right) d^{2}\left(x_{n_{l}}, T^{n_{l}} x_{n_{l}}\right) \\
& \quad=\sum_{m=n_{1}}^{n_{i}} \alpha_{m} \beta_{m}\left(1-\beta_{m}-a_{m} \beta_{m}-L^{2} \beta_{m}^{2}\right) d^{2}\left(x_{m}, T^{m} x_{m}\right) \\
& \leq \sum_{m=n_{1}}^{n_{i}}\left(a_{m}-1\right) M+d^{2}\left(x_{n_{1}}, p\right)-d^{2}\left(x_{n_{i}+1}, p\right) .
\end{aligned}
$$

From (57)-(60) and $\varepsilon \leq \alpha_{n} \leq \beta_{n}$, we obtain

$$
i \cdot \varepsilon^{2} \cdot \frac{D}{2} \cdot \varepsilon_{0} \leq \sum_{m=n_{1}}^{n_{i}}\left(a_{m}-1\right) M+d^{2}\left(x_{n_{1}}, p\right)-d^{2}\left(x_{n_{i}+1}, p\right) .
$$

Since $\sum_{n=1}^{\infty}\left(a_{n}-1\right)<\infty$ and the boundedness of $C$, the right side of (61) is bounded. However, if we have $i \rightarrow \infty$, then the left side of (61) is unbounded. This is a contradiction. Therefore,

$$
\lim _{n \rightarrow \infty} d\left(x_{n}, T^{n} x_{n}\right)=0
$$

Since $T$ is a uniformly $L$-Lipschitzian, from Lemma 6, we get

$$
\lim _{n \rightarrow \infty} d\left(x_{n}, T x_{n}\right)=0 \text {. }
$$

This completes the proof of Lemma 10.

Theorem 11. Let $(X, d)$ be a complete $C A T(0)$ space, let $C$ be a nonempty bounded closed convex subset of $X$, and let $T: C \rightarrow$ $C$ be a completely continuous and uniformly L-Lipschitzian and asymptotically hemicontractive with sequence $\left\{a_{n}\right\} \subset[1, \infty)$ satisfying $\sum_{n=1}^{\infty}\left(a_{n}-1\right)<\infty$, for all $n \in \mathbb{N}$. Given $x_{1} \in C$, define the iterative scheme $\left\{x_{n}\right\}$ by

$$
\begin{gathered}
x_{n+1}=\left(1-\alpha_{n}\right) x_{n} \oplus \alpha_{n} T^{n} y_{n}, \\
y_{n}=\left(1-\beta_{n}\right) x_{n} \oplus \beta_{n} T^{n} x_{n}, \quad n \geq 1 .
\end{gathered}
$$

If $\left\{\alpha_{n}\right\},\left\{\beta_{n}\right\} \subset[0,1]$ with $\varepsilon \leq \alpha_{n} \leq \beta_{n} \leq b$ for some $\varepsilon>0$ and $b \in\left(0,\left(\sqrt{1+L^{2}}-1\right) / L^{2}\right)$, then $\left\{x_{n}\right\}$ converges strongly to some fixed point of $T$.

Proof. Since $T$ is a completely continuous mapping in a bounded closed convex subset $C$ of complete metric space, from Schauder's theorem, $F(T)$ is nonempty. Since $T$ is completely continuous, there exist a convergent subset $\left\{T x_{n_{i}}\right\}$ of $\left\{T x_{n}\right\}$. Let

$$
\lim _{i \rightarrow \infty} T x_{n_{i}}=p
$$

Since $\lim _{n \rightarrow \infty} d\left(x_{n}, T x_{n}\right)=0$, from Lemma 10, we have

$$
\lim _{i \rightarrow \infty} x_{n_{i}}=p
$$

On the other hand, from the continuity of $T$, (66), and Lemma 10, we have

$$
d(p, T p)=\lim _{i \rightarrow \infty} d\left(x_{n_{i}}, T x_{n_{i}}\right)=0 .
$$

This means that $p$ is a fixed point of T. From (55), (57), and $\alpha_{n} \leq \beta_{n}$, we obtain Lemma 9 that

$$
d^{2}\left(x_{n+1}, p\right) \leq d^{2}\left(x_{n}, p\right)+\left(a_{n}-1\right) M
$$

From (66), there exists a subsequence $\left\{d^{2}\left(x_{n_{i}}, p\right)\right\}$ of $\left\{d^{2}\left(x_{n}, p\right)\right\}$ which converges to 0 . Therefore, from Lemma 5 and (68),

$$
\lim _{n \rightarrow \infty} d^{2}\left(x_{n}, p\right)=0
$$

Hence,

$$
\lim _{n \rightarrow \infty} x_{n}=p
$$

This completes the proof of Theorem 11. 
Corollary 12. Let $(X, d)$ be a complete CAT(0) space, let $C$ be a nonempty bounded closed convex subset of $X$, and let $T: C \rightarrow C$ be a completely continuous and uniformly L-Lipschitzian and asymptotically pseudocontractive with sequence $\left\{a_{n}\right\} \subset[1, \infty)$ satisfying $\sum_{n=1}^{\infty}\left(a_{n}^{2}-1\right)<\infty$, for all $n \in \mathbb{N}$. Given $x_{1} \in C$, define the iterative scheme $\left\{x_{n}\right\}$ by

$$
\begin{gathered}
x_{n+1}=\left(1-\alpha_{n}\right) x_{n} \oplus \alpha_{n} T^{n} y_{n}, \\
y_{n}=\left(1-\beta_{n}\right) x_{n} \oplus \beta_{n} T^{n} x_{n}, \quad n \geq 1 .
\end{gathered}
$$

If $\left\{\alpha_{n}\right\},\left\{\beta_{n}\right\} \subset[0,1]$ with $\varepsilon \leq \alpha_{n} \leq \beta_{n} \leq b$ for some $\varepsilon>0$ and $b \in\left(0,\left(\sqrt{1+L^{2}}-1\right) / L^{2}\right)$, then $\left\{x_{n}\right\}$ converges strongly to some fixed point of $T$.

Proof. By Definition 2, $T$ is an asymptotically pseudocontractive mapping, then $T$ is an asymptotically hemicontractive mapping. Since $a_{n} \in[1, \infty)$, we have $a_{n}^{2}-1 \geq a_{n}-1 \geq 0$. Obviously, $\sum_{n=1}^{\infty}\left(a_{n}-1\right) \leq \sum_{n=1}^{\infty}\left(a_{n}^{2}-1\right)<\infty$. Therefore, Corollary 12 can be proved by using Theorem 11 .

\section{Some Remarks and Open Problems}

Let $S$ be a semigroup. We denote by $B(S)$ the space of all bounded real-valued functions defined on $S$ with supremum norm. For each $s \in S$, we define the left and right translation operators $l_{s}$ and $r_{s}$ on $B(S)$ by

$$
\left(l_{s} f\right)(t)=f(s t), \quad\left(r_{s} f\right)(t)=f(t s),
$$

for each $t \in S$ and $f \in B(S)$, respectively. Let $X$ be a subspace of $B(S)$ containing 1 . An element $\mu$ in the dual space $X^{*}$ of $X$ is said to be a mean on $X$ if $\|\mu\|=\mu(1)=1$. For $s \in S$, we can define a point evaluation $\delta_{s}$ by $\delta_{s}(f)=f(s)$ for each $f \in X$. It is well known that $\mu$ is mean on $X$ if and only if

$$
\inf _{s \in S} f(s) \leq \mu(f) \leq \sup _{s \in S} f(s)
$$

for each $f \in X$. Each mean on $X$ is the weak ${ }^{*}$-limit of convex combination of point evaluations.

Let $X$ be a translation invariant subspace of $B(S)$ (i.e., $l_{s} X \subset X$ and $r_{s} X \subset X$ for each $s \in S$ ) containing 1 . Then a mean $\mu$ on $X$ is said to be left invariant (resp., right invariant) if

$$
\mu\left(l_{s} f\right)=\mu(f) \quad\left(\text { resp., } \mu\left(r_{s} f\right)=\mu(f)\right),
$$

for each $s \in S$ and $f \in X$. A mean $\mu$ on $X$ is said to be invariant if $\mu$ is both left and right invariant ([30-34]). $X$ is said to be left (resp., right) amenable if $X$ has a left (resp., right) invariant mean. $X$ is amenable if $X$ is left and right amenable. In this case, we say that the semigroup $S$ is an amenable semigroup (see $[35,36]$ ). Moreover, $B(S)$ is amenable when $S$ is a commutative semigroup or a solvable group. However, the free group or semigroup of two generators is not left or right amenable.

A net $\left\{\mu_{\alpha}\right\}$ of means on $X$ is said to be asymptotically left (resp., right) invariant if

$$
\begin{aligned}
& \lim _{\alpha}\left(\mu_{\alpha}\left(l_{s} f\right)-\mu_{\alpha}(f)\right)=0 \\
& \text { (resp., } \left.\lim _{\alpha}\left(\mu_{\alpha}\left(r_{s} f\right)-\mu_{\alpha}(f)\right)=0\right),
\end{aligned}
$$

for each $f \in X$ and $s \in S$, and it is said to be left (resp., right) strongly asymptotically invariant (or strong regular) if

$$
\left.\lim _{\alpha}\left\|l_{s}^{*} \mu_{\alpha}-\mu_{\alpha}\right\|=0 \quad \text { (resp., } \lim _{\alpha}\left\|r_{s}^{*} \mu_{\alpha}-\mu_{\alpha}\right\|=0\right),
$$

for each $s \in S$, where $l_{s}^{*}$ and $r_{s}^{*}$ are the adjoint operators of $l_{s}$ and $r_{s}$, respectively. Such nets were first studied by Day in [35] where they were called weak* invariant and norm invariant, respectively.

It is easy to see that if a semigroup $S$ is left (resp., right) amenable, then the semigroup $S^{\prime}=S \cup\{e\}$, where es ${ }^{\prime}=$ $s^{\prime} e=s^{\prime}$ for all $s^{\prime} \in S$ is also left (resp., right) amenable and conversely.

A semigroup $S$ is called left reversible if any two right ideals of $S$ have nonvoid intersection, that is, $a S \cap b S \neq \emptyset$ for $a, b \in S$. In this case, $(S, \preceq)$ is a directed system when the binary relation " $\preceq$ " on $S$ is defined by $a \preceq b$ if and only if $\{a\} \cup a S \supseteq\{b\} \cup b S$ for $a, b \in S$. It is easy to see that $t \preceq t s$ for all $t, s \in S$. Further, if $t \preceq s$, then $p t \preceq p s$ for all $p \in S$. The class of left reversible semigroup includes all groups and commutative semigroups. If a semigroup $S$ is left amenable, then $S$ is left reversible. But the converse is not true ([31, 3741]).

Let $S$ be a semigroup and $F(T)$ denote the fixed point set of $T$. Then $\mathfrak{J}=\left\{T_{s}: s \in S\right\}$ is called a representation of $S$ if $T_{e}=I$ and $T_{s t}=T_{s} T_{t}$ for each $s, t \in S$. We denote by $F(\Im)$ the set of common fixed points of $\left\{T_{s}: s \in S\right\}$, that is,

$$
F(\Im)=\bigcap_{s \in S} F\left(T_{s}\right)=\bigcap_{s \in S}\left\{x \in C: T_{s} x=x\right\} .
$$

Open Problem 1. It will be interesting to obtain a generalization of both Theorems 7 and 11 to commutative, amenable, and reversible semigroups as in the case of Hilbert spaces or some Banach spaces ( $c f .[8,30,32,42-45])$.

For a real number $\kappa$, a $C A T(\kappa)$ space is defined by a geodesic metric space whose geodesic triangle is sufficiently thinner than the corresponding triangle in a model space with curvature $\kappa$.

For $\kappa=0$, the 2-dimensional model space $M_{\kappa}^{2}=M_{0}^{2}$ is the Euclidean space $\mathbb{R}^{2}$ with the metric induced from the Euclidean norm. For $\kappa>0, M_{\mathcal{\kappa}}^{2}$ is the 2-dimensional sphere $(1 / \sqrt{\kappa}) \mathbb{S}^{2}$ whose metric is length of a minimal great arc joining each two points. For $\kappa<0, M_{\kappa}^{2}$ is the 2dimensional hyperbolic space $(1 / \sqrt{-\kappa}) \mathbb{M}^{2}$ with the metric defined by a usual hyperbolic distance. For more details about the properties of $C A T(\kappa)$ spaces, see $[4,46-48]$.

Open Problem 2. It will be interesting to obtain a generalization of both Theorems 7 and 11 to $C A T(\kappa)$ space.

\section{Conflict of Interests}

The author declares that there is no conflict of interests regarding the publication of this paper.

\section{Acknowledgments}

The author would like to thank Professor A. T.-M Lau and Professor J. K. Kim for their helpful suggestions. Also, special 
thanks are due to the referees for their deep insight which improved the presentation of this paper. This research was supported by the Basic Science Research Program through the National Research Foundation of Korea (NRF) funded by the Ministry of Education (2012R1A1A4A01010526).

\section{References}

[1] W. Takahashi, "A convexity in metric space and nonexpansive mappings. I," Kōdai Mathematical Seminar Reports, vol. 22, pp. 142-149, 1970.

[2] J. K. Kim, K. S. Kim, and Y. M. Nam, "Convergence and stability of iterative processes for a pair of simultaneously asymptotically quasi-nonexpansive type mappings in convex metric spaces," Journal of Computational Analysis and Applications, vol. 9, no. 2, pp. 159-172, 2007.

[3] M. Gromov, "Hyperbolic groups," in Essays in Group Theory, vol. 8 of Publications of the Research Institute for Mathematical Sciences, pp. 75-263, Springer, New York, NY, USA, 1987.

[4] M. R. Bridson and A. Haefliger, Metric Spaces of Non-Positive Curvature, Springer, Berlin, Germany, 1999.

[5] D. Burago, Y. Burago, and S. Ivanov, A Course in Metric Geometry, vol. 33 of Graduate Studies in Mathematics, American Mathematical Society, Providence, RI, USA, 2001.

[6] W. A. Kirk, "Fixed point theorems in $C A T(0)$ spaces and $R-$ trees," Fixed Point Theory and Applications, no. 4, pp. 309-316, 2004.

[7] F. Bruhat and J. Tits, "Groupes réductifs sur un corps local. I. Données radicielles valuées," Institut des Hautes Études Scientifiques. Publications Mathématiques, no. 41, pp. 5-251, 1972.

[8] M. A. Khamsi and W. A. Kirk, "On uniformly Lipschitzian multivalued mappings in Banach and metric spaces," Nonlinear Analysis. Theory, Methods \& Applications, vol. 72, no. 3-4, pp. 2080-2085, 2010.

[9] S. Dhompongsa and B. Panyanak, "On $\Delta$-convergence theorems in CAT(0) spaces," Computers \& Mathematics with Applications, vol. 56, no. 10, pp. 2572-2579, 2008.

[10] Q. Liu, "Convergence theorems of the sequence of iterates for asymptotically demicontractive and hemicontractive mappings," Nonlinear Analysis. Theory, Methods \& Applications, vol. 26, no. 11, pp. 1835-1842, 1996.

[11] J. Schu, "Iterative construction of fixed points of asymptotically nonexpansive mappings," Journal of Mathematical Analysis and Applications, vol. 158, no. 2, pp. 407-413, 1991.

[12] P. Chaoha and A. Phon-on, "A note on fixed point sets in CAT(0) spaces," Journal of Mathematical Analysis and Applications, vol. 320, no. 2, pp. 983-987, 2006.

[13] C. E. Chidume, "Convergence theorems for asymptotically pseudocontractive mappings," Nonlinear Analysis. Theory, Methods \& Applications, vol. 49, no. 1, pp. 1-11, 2002.

[14] X. P. Ding, "Iteration processes for nonlinear mappings in convex metric spaces," Journal of Mathematical Analysis and Applications, vol. 132, no. 1, pp. 114-122, 1988.

[15] K. Goebel and S. Reich, Uniformly Convexity, Hyperbolic Geometry, and Nonexpansive Mappings, vol. 83 of Monographs and Textbooks in Pure and Applied Mathematics, Marcel Dekker, New York, NY, USA, 1984.

[16] J.-C. Huang, "Implicit iteration process for a finite family of asymptotically hemi-contractive mappings in Banach spaces," Nonlinear Analysis. Theory, Methods \& Applications, vol. 66, no. 9, pp. 2091-2097, 2007.
[17] J. K. Kim, K. S. Kim, and S. M. Kim, "Convergence theorems of implicit iteration process for a finite family of asymptotically quasi-nonexpansive mappings in convex metric spaces," RIMS Kokyuroku, vol. 1484, pp. 40-51, 2006.

[18] J. K. Kim, K. H. Kim, and K. S. Kim, "Convergence theorems of modified three-step iterative sequences with mixed errors for asymptotically quasi-nonexpansive mappings in Banach spaces," Panamerican Mathematical Journal, vol. 14, no. 1, pp. 45-54, 2004.

[19] J. K. Kim, K. H. Kim, and K. S. Kim, "Three-step iterative sequences with errors for asymptotically quasi-nonexpansive mappings in convex metric spaces," RIMS Kokyuroku, vol. 1365, pp. 156-165, 2004.

[20] U. Kohlenbach and L. Leuştean, "Mann iterates of directionally nonexpansive mappings in hyperbolic spaces," Abstract and Applied Analysis, no. 8, pp. 449-477, 2003.

[21] L. Leustean, "A quadratic rate of asymptotic regularity for CAT(0)-spaces," Journal of Mathematical Analysis and Applications, vol. 325, no. 1, pp. 386-399, 2007.

[22] C. Moore and B. V. C. Nnoli, "Iterative sequence for asymptotically demicontractive maps in Banach spaces," Journal of Mathematical Analysis and Applications, vol. 302, no. 2, pp. 557$562,2005$.

[23] M. O. Osilike, A. Udomene, D. I. Igbokwe, and B. G. Akuchu, "Demiclosedness principle and convergence theorems for $k$ strictly asymptotically pseudocontractive maps," Journal of Mathematical Analysis and Applications, vol. 326, no. 2, pp. 1334-1345, 2007.

[24] S. Reich and I. Shafrir, "Nonexpansive iterations in hyperbolic spaces," Nonlinear Analysis. Theory, Methods \& Applications, vol. 15, no. 6, pp. 537-558, 1990.

[25] S. Saejung, "Halpern's iteration in CAT(0) spaces," Fixed Point Theory and Applications, vol. 2010, Article ID 471781, 13 pages, 2010.

[26] C. Wang and L.-W. Liu, "Convergence theorems for fixed points of uniformly quasi-Lipschitzian mappings in convex metric spaces," Nonlinear Analysis. Theory, Methods \& Applications, vol. 70, no. 5, pp. 2067-2071, 2009.

[27] H. Zhou and Y. Su, "Strong convergence theorems for a family of quasi-asymptotic pseudo-contractions in Hilbert spaces," Nonlinear Analysis. Theory, Methods \& Applications, vol. 70, no. 11, pp. 4047-4052, 2009.

[28] S. Ishikawa, "Fixed points by a new iteration method," Proceedings of the American Mathematical Society, vol. 44, pp. 147-150, 1974.

[29] W. R. Mann, “Mean value methods in iteration," Proceedings of the American Mathematical Society, vol. 4, pp. 506-510, 1953.

[30] K. S. Kim, "Ergodic theorems for reversible semigroups of nonlinear operators," Journal of Nonlinear and Convex Analysis, vol. 13, no. 1, pp. 85-95, 2012.

[31] A. T.-M. Lau, "Invariant means on almost periodic functions and fixed point properties," The Rocky Mountain Journal of Mathematics, vol. 3, pp. 69-76, 1973.

[32] A. T.-M. Lau, "Semigroup of nonexpansive mappings on a Hilbert space," Journal of Mathematical Analysis and Applications, vol. 105, no. 2, pp. 514-522, 1985.

[33] A. T.-M. Lau, "Invariant means and fixed point properties of semigroup of nonexpansive mappings," Taiwanese Journal of Mathematics, vol. 12, no. 6, pp. 1525-1542, 2008. 
[34] A. T.-M. Lau and W. Takahashi, "Invariant means and fixed point properties for non-expansive representations of topological semigroups," Topological Methods in Nonlinear Analysis, vol. 5, no. 1, pp. 39-57, 1995.

[35] M. M. Day, “Amenable semigroups," Illinois Journal of Mathematics, vol. 1, pp. 509-544, 1957.

[36] A. T.-M. Lau, N. Shioji, and W. Takahashi, "Existence of nonexpansive retractions for amenable semigroups of nonexpansive mappings and nonlinear ergodic theorems in Banach spaces," Journal of Functional Analysis, vol. 161, no. 1, pp. 62-75, 1999.

[37] R. D. Holmes and A. T.-M. Lau, "Non-expansive actions of topological semigroups and fixed points," Journal of the London Mathematical Society, vol. 5, no. 2, pp. 330-336, 1972.

[38] K. S. Kim, "Nonlinear ergodic theorems of nonexpansive type mappings," Journal of Mathematical Analysis and Applications, vol. 358, no. 2, pp. 261-272, 2009.

[39] A. T.-M. Lau and P. F. Mah, "Fixed point property for Banach algebras associated to locally compact groups," Journal of Functional Analysis, vol. 258, no. 2, pp. 357-372, 2010.

[40] A. T.-M. Lau and W. Takahashi, "Weak convergence and nonlinear ergodic theorems for reversible semigroups of nonexpansive mappings," Pacific Journal of Mathematics, vol. 126, no. 2, pp. 277-294, 1987.

[41] A. T.-M. Lau and Y. Zhang, "Fixed point properties of semigroups of non-expansive mappings," Journal of Functional Analysis, vol. 254, no. 10, pp. 2534-2554, 2008.

[42] K. S. Kim, "Convergence of a hybrid algorithm for a reversible semigroup of nonlinear operators in Banach spaces," Nonlinear Analysis. Theory, Methods \& Applications, vol. 73, no. 10, pp. 3413-3419, 2010.

[43] S. Saewan and P. Kumam, "Explicit iterations for Lipschitzian semigroups with the Meir-Keeler type contraction in Banach spaces," Journal of Inequalities and Applications, vol. 2012, article 279, 2012.

[44] Y. Song and S. Xu, "Strong convergence theorems for nonexpansive semigroup in Banach spaces," Journal of Mathematical Analysis and Applications, vol. 338, no. 1, pp. 152-161, 2008.

[45] C. Wu, S. Y. Cho, and M. Shang, "Moudafi's viscosity approximations with demi-continuous and strong pseudo-contractions for non-expansive semigroups," Journal of Inequalities and Applications, vol. 2010, Article ID 645498, 10 pages, 2010.

[46] R. Espínola and A. Fernández-León, "CAT( $($ )-spaces, weak convergence and fixed points," Journal of Mathematical Analysis and Applications, vol. 353, no. 1, pp. 410-427, 2009.

[47] Y. Kimura and K. Satô, "Convergence of subsets of a complete geodesic space with curvature bounded above," Nonlinear Analysis. Theory, Methods \& Applications, vol. 75, no. 13, pp. 5079-5085, 2012.

[48] Y. Kimura and K. Satô, "Halpern iteration for strongly quasinonexpansive mappings on a geodesic space with curvature bounded above by one," Fixed Point Theory and Applications, vol. 2013, article 7, 2013. 


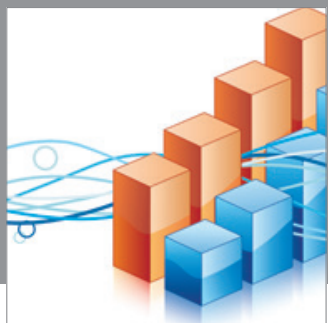

Advances in

Operations Research

mansans

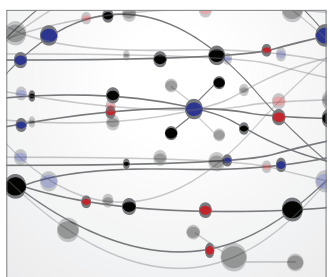

The Scientific World Journal
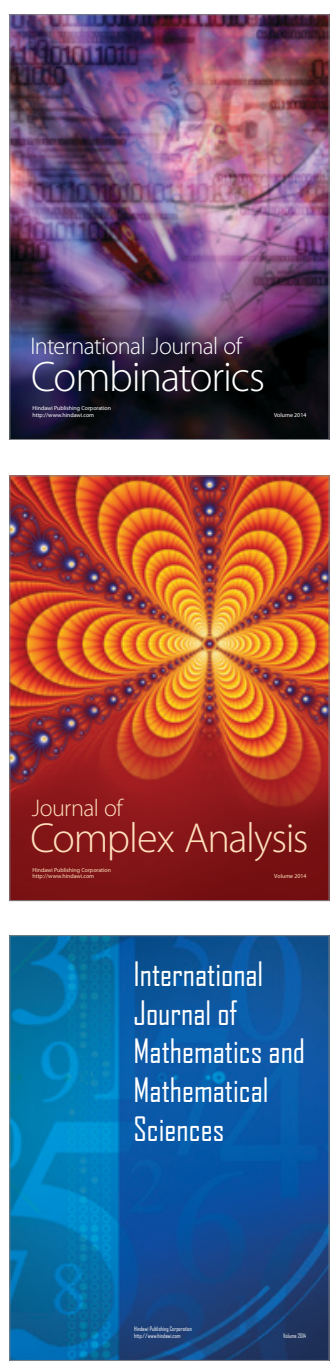
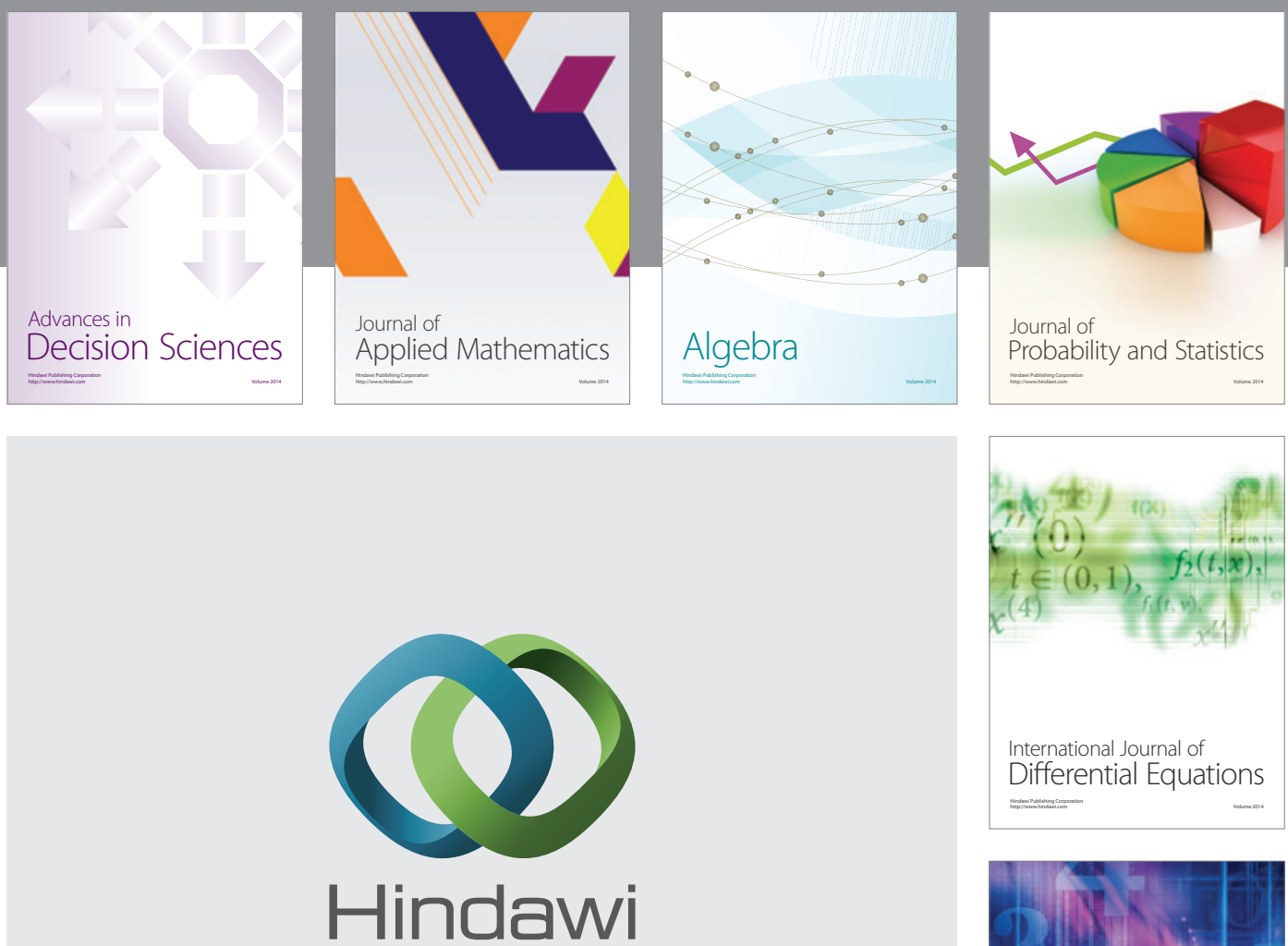

Submit your manuscripts at http://www.hindawi.com
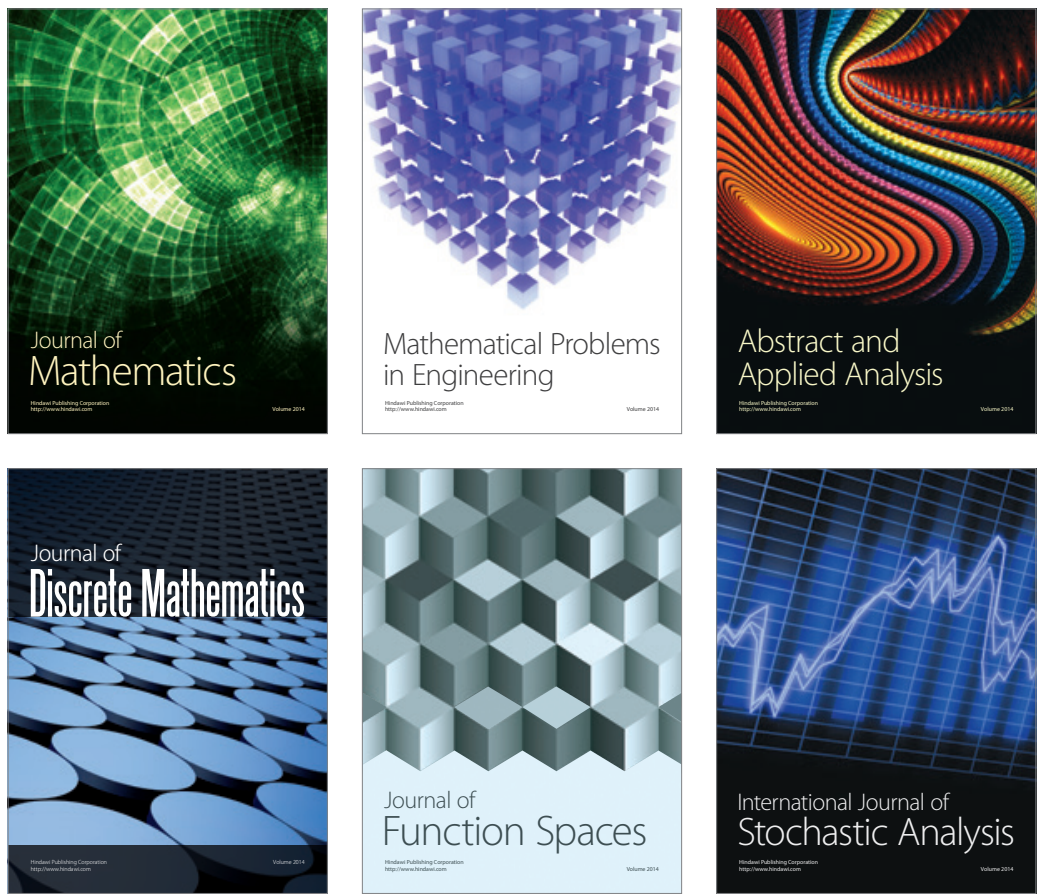

Journal of

Function Spaces

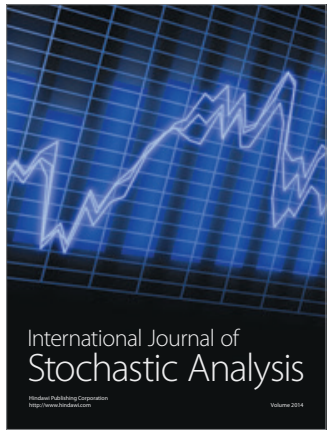

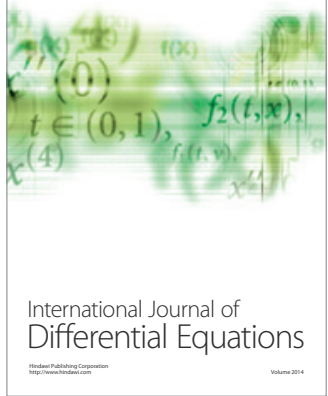
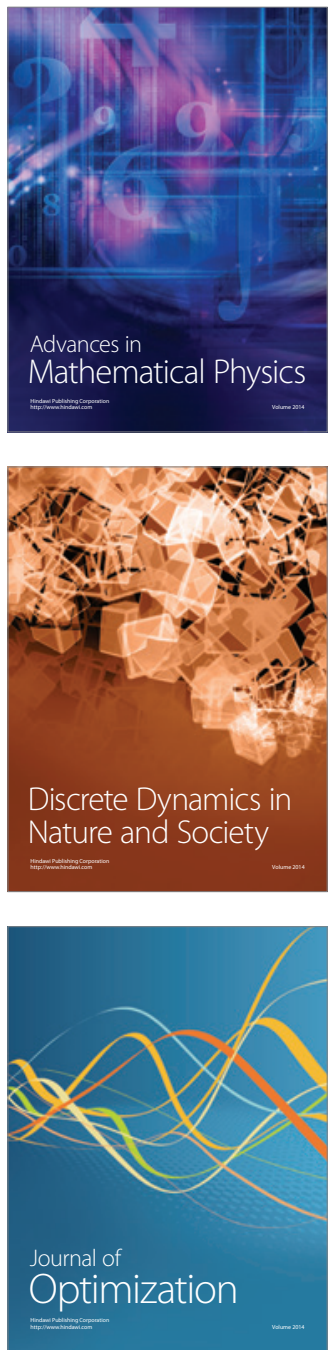